\title{
A New Method in the Combinatorics of the Topological Expansion
}

D. Bessis

Service de Physique Théorique, CEN Saclay, F-91190 Gif-sur-Yvette, France

\begin{abstract}
We reduce the counting problem for the vacuum diagrams of a $\phi^{4}$ theory to a moment problem. As a consequence we are able to give the generating function for the counting of diagrams on a torus with one hole, besides the known result for planar diagrams. The method can be extended to $\phi^{n}$ theory and also to the counting of diagrams on a torus with an arbitrary number of holes.
\end{abstract}

\section{Introduction}

In their paper Planar Diagrams [1], E. Brézin, C. Itzykson, C. Parisi and J.B. Zuber have discussed the combinatorics of Quartic Vertices, and found the generating function $E^{(0)}(g)$ which solves the counting problem for the vacuum diagrams in the planar approximation. The technic used was the saddle point method. Unfortunately this method does not provide an easy way to reach even the next generating function $E^{(1)}(g)$ which solves the counting problem on a torus with one hole.

In this paper, we have obtained for this generating function a rather simple expression:

$$
E^{(1)}(g)=\frac{1}{12} \log \left(2-a^{2}\right)
$$

with, following the notation in [1]:

$$
12 g a^{4}+a^{2}-1=0 \text {, }
$$

where the root to be taken (I.2) is the root regular at $g=0$. Of course we have also verified that the generating function $E^{(0)}(g)$ is given by:

$$
E^{(0)}(g)=-\frac{1}{2} \log a^{2}+\frac{1}{24}\left(a^{2}-1\right)\left(9-a^{2}\right) .
$$

The general case, the computation of $E^{(k)}(g)$ for $k \geqq 2$ will be considered elsewhere.

As shown in [1], the generating functions $E^{(k)}(g)$ appear as coefficients in the asymptotic expansion:

$$
-\frac{1}{n^{2}} \log \frac{I_{n}(g / n)}{I_{n}(0)}=E_{0}(g)+\frac{E_{1}(g)}{n^{2}}+\frac{E_{2}(g)}{n^{4}}+\ldots
$$


where $I_{n}(g / n)$ is the integral:

$$
I_{n}(g / n)=\int_{-\infty}^{+\infty} \prod_{1 \leqq i \leqq j \leqq n}\left(\lambda_{i}-\lambda_{j}\right)^{2} \prod_{i=1}^{i=n} e^{-(1 / 2) \lambda_{l}^{2}-(g / n) \lambda_{\imath}^{4}} d \lambda_{i}
$$

We show that $I_{n}$ can be thought as the Hadamard determinant of order $n$ associated with the positive measure

$$
d \mu(\lambda)=e^{-\lambda^{2} / 2-(g / n) \lambda^{4}} d \lambda
$$

By expanding in continued fraction the generating function of the moments associated with the measure (I.6) we obtain a non linear recursive relation for the coefficients of the continued fractions.

This relation is equivalent to a non linear recursive relation among 6 consecutive $I_{n}$.

We show that, in the limit $n \rightarrow \infty$, those recursive relations transform into an infinite set of coupled differential equations, forming a triangular system which can be analysed.

The method extends naturally to measures which are exponentials of an arbitrary polynomial, and therefore to vertices with $n$ lines.

Here we have only worked out the functions $E^{(0)}(g)$ and $E^{(1)}(g)$ but, it seems feasible with this method to get eventually $E^{(k)}$ in closed form: this will be discussed in a near future.

\section{The Moment Problem Formulation}

Let us consider a positive measure $d \mu(x)$ defined on $[-\infty,+\infty]$, and its associated moments:

$$
\mu_{k}=\int_{-\infty}^{+\infty} x^{k} d \mu(x) \quad k=0,1,2, \ldots
$$

The integral

$$
I_{n}=\int_{-\infty}^{+\infty} \prod_{i=1}^{i=n} d \mu\left(x_{i}\right) \prod_{1 \leqq l<j \geqq n}\left(x_{i}-x_{j}\right)^{2}
$$

can be reexpressed in terms of the $\mu_{k}$ 's, by the formula (see Appendix AI)

$$
I_{n}=n ! D_{n-1},
$$

where $D_{n}$ is the Hadamard determinant of order $(n+1)$, associated with the moments $\mu_{k}$ :

$$
D_{n}=\left|\begin{array}{cccc}
\mu_{0} & \mu_{1} & \ldots & \mu_{n} \\
\mu_{1} & \mu_{2} & \ldots & \mu_{n+1} \\
\ldots & \ldots & \ldots & \ldots \\
\mu_{n} & \mu_{n+1} & \ldots & \mu_{2 n}
\end{array}\right|
$$


The generating function of the $\mu_{k}$ 's, has, for formal expansion in powers of $1 / z$ :

$$
G(z)=\frac{\mu_{0}}{z}+\frac{\mu_{1}}{z^{2}}+\ldots+\frac{\mu_{k}}{z^{k+1}}+\ldots
$$

We shall normalize the problem, by setting:

$$
\bar{G}(z)=\frac{G(z)}{\mu_{0}}=\frac{s_{0}}{z}+\frac{s_{1}}{z^{2}}+\ldots+\frac{s_{k}}{z^{k+1}}+\ldots
$$

with

$$
\left\{\begin{array}{l}
s_{0}=+1 \\
s_{k}=\frac{\mu_{k}}{\mu_{0}}
\end{array}\right.
$$

to $\bar{G}(z)$ is associated the Hadamard determinant:

$$
\begin{gathered}
\bar{D}_{n}=\left|\begin{array}{llll}
s_{0} & s_{1} & \ldots & s_{n} \\
s_{1} & s_{2} & \ldots & s_{n+1} \\
\ldots & \ldots & \ldots & \ldots \\
s_{n} & s_{n+1} & \ldots & s_{2 n}
\end{array}\right| \\
D_{n} \text { and } \bar{D}_{n} \text { are connected by: } \\
D_{n}=\mu_{0}^{n+1} \bar{D}_{n} .
\end{gathered}
$$

We shall, from now on, consider the case where the odd moments $\mu_{2 p+1}$ are identically zero, and therefore introduce the continued fraction expansion of $\bar{G}(z)[2]:$

$$
\bar{G}(z)=\frac{1}{z-\frac{R_{1}}{z-\frac{R_{2}}{z-\frac{R_{3}}{z-}}}}
$$

The $n$th approximation to it, is a rational fraction, the $[n-1 / n](z)$ Padé Approximation, that we write:

$$
[n-1 / n](z)=\frac{N_{n}(z)}{M_{n}(z)},
$$

where

$$
M_{n}(z)=z^{n}+\ldots,
$$

is a polynomial of degree $n$ in $z$ with the highest degree coefficient normalized to 1 , and

$$
N_{n}(z)=z^{n-1}+\ldots,
$$


is a polynomial of degree $(n-1)$ in $z$ with the highest degree coefficient normalized to 1 .

The first approximations read:

$$
\begin{aligned}
& {[0 / 1]=\frac{1}{z}} \\
& {[1 / 2]=\frac{1}{z-\frac{R_{1}}{z}}} \\
& {[2 / 3]=\frac{1}{z-\frac{R_{1}}{z-\frac{R_{2}}{z}}}} \\
& \left.\begin{array}{l}
N_{1}=1 \\
M_{1}=z
\end{array}\right\} \\
& \left.\begin{array}{l}
N_{2}=z \\
M_{2}=z^{2}-R_{1} \\
R_{1}=s_{2}
\end{array}\right\} \\
& N_{3}=z^{2}-R_{2} \\
& M_{3}=z\left[z^{2}-\left(R_{1}+R_{2}\right)\right] \\
& R_{2}=\frac{s_{4}-s_{2}^{2}}{s_{2}}
\end{aligned}
$$

and

$$
[3 / 4]=\frac{1}{z-\frac{R_{1}}{z-\frac{R_{2}}{z-\frac{R_{3}}{z}}}}
$$

$$
\begin{aligned}
& N_{4}=z\left[z^{2}-\left(R_{2}+R_{3}\right)\right] \\
& M_{4}=z^{4}-z^{2}\left(R_{1}+R_{2}+R_{3}\right)+R_{1} R_{3} \\
& R_{3}=\frac{s_{2} s_{6}-s_{4}^{2}}{s_{2}\left(s_{4}-s_{2}^{2}\right)}
\end{aligned}
$$

From $\bar{D}_{n}=R_{n} R_{n-1}^{2} \ldots R_{1}^{n-1}$ (II.22), we see that:

$$
\begin{aligned}
& \bar{D}_{1}=R_{1}=s_{2} \\
& \bar{D}_{2}=R_{2} R_{1}^{2}=\left(s_{4}-s_{2}^{2}\right) s_{2} \\
& \bar{D}_{3}=R_{3} R_{2}^{2} R_{1}^{3}=\left(s_{6} s_{2}-s_{4}^{2}\right)\left(s_{4}-s_{2}^{2}\right) .
\end{aligned}
$$

1 This is a consequence of $R_{n} \bar{D}_{n-1}^{2}=\bar{D}_{n-2} \bar{D}_{n}[13]$ 
Finally, combining (II.3), (II.9) and (II.22), we get:

$$
I_{n}=n ! \mu_{0}^{n} R_{1}^{n-1} R_{2}^{n-2} \ldots R_{n-2}^{2} R_{n-1} .
$$

The problem of evaluating $I_{n}$, is therefore reduced to the problem of computing the $R_{n}$ from the measure $d \mu(x)$.

To end this paragraph we give for completeness the recursive relation among the polynomials $M_{n}(z)$ or $N_{n}(z)$, the numerators and denominators of the $n$th approximation fulfil the relation:

$$
Y_{k+1}(z)=z Y_{k}(z)-R_{k} Y_{k-1}(z),
$$

with

$$
\begin{array}{ll}
N_{0}(z)=0 & N_{1}(z)=1 \\
M_{0}(z)=1 & M_{1}(z)=z .
\end{array}
$$

As is well known the $M_{n}(z)$ form the set of orthogonal polynomial with respect to the measure $d \mu(x)$.

\section{Asymptotic Formulae}

In the present situation the measure $d \mu(x)$ reads:

$$
d \mu(x)=e^{-(1 / 2) x^{2}-\beta x^{4}},
$$

with

$$
\beta=\frac{g}{n} \text {. }
$$

The moments of the measure:

$$
\mu_{k}=\int_{-\infty}^{+\infty} x^{k} e^{-x^{2} / 2-\beta x^{4}} d x
$$

have the following properties:

$$
\begin{aligned}
& \mu_{2 k+1}=0 \\
& (2 k+1) \mu_{2 k}=\mu_{2 k+2}+4 \beta \mu_{2 k+4}
\end{aligned}
$$

or

$$
\left.\begin{array}{l}
s_{2 k+1}=0 \\
(2 k+1) s_{2 k}=s_{2 k 2}+4 \beta s_{2 k+4}
\end{array}\right\}
$$

The recursive relation (III.4) shows clearly that $G(z)$, the generating function of the moments has to be the solution of a linear first order differential equation with polynomials coefficients. We shall use this fundamental remark in Sect. IV.

Expanding for $k$ even (III.3) in powers of $\beta$, we get:

$$
\mu_{2 k}\left(\frac{g}{n}\right)=\sum_{p=0}^{\infty} \frac{\left(-\frac{g}{n}\right)^{p}}{p !} 2^{k+2 p+1 / 2} \Gamma(k+2 p+1 / 2)
$$




$$
=\sqrt{2 \pi} \sum_{p=0}^{\infty} \frac{\left(-\frac{g}{n}\right)^{p}}{p !} 1.3 .5 \ldots(2 k+4 p-1) .
$$

In particular:

$$
\bar{\mu}_{0}(g / n)=\frac{\mu_{0}(g / n)}{\mu_{0}(0)}=\sum_{p=0}^{\infty} \frac{\left(-\frac{g}{n}\right)^{p}}{p !} 1.3 .5 \ldots(4 p-1) .
$$

Following reference [1], we want to evaluate the asymptotic expansion:

$$
\frac{1}{n^{2}} \log \frac{I_{n}(g / n)}{I_{n}(0)}=-E_{0}(g)-\frac{E_{1}(g)}{n^{2}}-\frac{E_{2}(g)}{n^{4}} \ldots
$$

Setting

$$
\bar{R}_{k}(g / n)=\frac{R_{k}(g / n)}{R_{k}(0)}
$$

we have, from formula (II.23):

$$
\frac{1}{n^{2}} \log \frac{I_{n}(g / n)}{I_{n}(0)}=\frac{1}{n} \log \bar{\mu}_{0}(g / n)+\frac{1}{n} \sum_{p=0}^{p=n}\left(1-\frac{p}{n}\right) \log \bar{R}_{p}(g / n),
$$

where we have set

$$
\bar{R}_{0}=1 \text {. }
$$

It will be shown in the sequel that $\bar{R}_{p}(g / n)$ has the following expansion:

$$
\bar{R}_{p}(g / n)=\rho(p / n)+\frac{1}{n^{2}} \rho_{2}(p / n)+\ldots
$$

with

$$
\rho(0)=1,
$$

where $\rho(x), \rho_{2}(x)$ are holomorphic on $[0,1]$.

By making use of the Euler formula, that we recall:

Let $f(x)$ be of class $C^{2 p}$ on $[0,1]$, then:

$$
\begin{aligned}
\frac{1}{n} \sum_{p=0}^{p=n} f(p / n)= & \int_{0}^{1} f(x) d x+\frac{1}{2 n}[f(0)+f(1)]+\frac{B_{1}}{2 !} \frac{1}{n^{2}}\left[f^{\prime}(1)-f^{\prime}(0)\right] \\
& -\frac{B_{2}}{4 !} \frac{1}{n^{4}}\left[f^{\prime \prime \prime}(1)-f^{\prime \prime \prime}(0)\right]+\ldots+(-)^{p} \frac{B_{p-1}}{(2 p-2) ! n^{2 p-2}} \\
& \cdot\left[f^{(2 p-3)}(1)-f^{(2 p-3)}(0)\right]+(-)^{p+1} \frac{B_{p}}{(2 p) ! \frac{1}{n^{2 p+1}}} \\
& \cdot\left[f^{(2 p)}\left(x_{1}\right)+f^{(2 p)}\left(x_{2}\right)+\ldots+f^{(2 p)}\left(x_{p}\right)\right]
\end{aligned}
$$

where

$$
\frac{i-1}{n}<x_{i}<\frac{i}{n}
$$


We get:

$$
-E_{0}(g)=\int_{0}^{1}(1-x) \log \rho(x) d x
$$

and

$$
-E_{1}(g)=-3 g+\frac{1}{12}\left[-\rho^{\prime}(0)-\log \rho(1)\right]+\int_{0}^{1}(1-x) \frac{\rho_{2}(x)}{\rho(x)} d x
$$

where we have used (III.6) to expand $\log \bar{\mu}_{0}(g / n)$ up to second order in $1 / n$, as well as (III.11).

\section{A Recursion Formula}

We shall now derive a recursion formula for the $R_{p}(\beta)$. In the sequel we shall keep $\beta$ fixed independent of $n$. We consider the generating function of the $\mu_{k}$ :

$$
G(z)=\sum_{k+0}^{\infty} \frac{\mu_{k}}{z^{k+1}}=\int_{-\infty}^{+\infty} \frac{e^{-x^{2} / 2-\beta x^{4}} d x}{z-x} .
$$

As pointed out in the previous section, $G(z)$ must fulfil a linear first order differential equation. It is not difficult to obtain the equation:

$$
G^{\prime}(z)+\left[z+4 \beta z^{3}\right] G(z)=\mu_{0}+4 \beta \mu_{2}+4 \beta \mu_{0} z^{2} .
$$

Therefore $\bar{G}(z)=G(z) / \mu_{0}$ satisfies the equation:

$$
W(z) \bar{G}^{\prime}(z)=2 V(z) \bar{G}(z)+U(z)
$$

where

$$
\begin{aligned}
& W(z)=1 \\
& V(z)=-\frac{z}{2}-2 \beta z^{3} \\
& U(z)=\left(1+4 \beta s_{2}\right)+4 \beta z^{2} .
\end{aligned}
$$

Here $s_{2}=\frac{\mu_{2}}{\mu_{0}}$ following (II.7).

We must now expand in continued fraction the solution of (IV.3) which admits the positivity representation (IV.1).

Following Laguerre [4] (we give in Appendix AII the necessary elements to understand this construction), we consider the function:

$$
V+\frac{n W}{z}=-2 \beta z^{3}-\frac{z}{2}+\frac{n}{z}
$$

to which is associated the polynomial $\Omega_{n}$ having the same term of highest degree as the polynomial part of the previous function:

$$
\Omega_{n}=-2 \beta z^{3}+\gamma_{n} z,
$$

where in $\Omega_{n}$ there is no even part due to a parity argument. We also introduce, 
still using the same parity argument:

$$
\begin{aligned}
& \theta_{n}=c_{n} z^{2}+d_{n} \\
& Q_{n}=z .
\end{aligned}
$$

The Laguerre equations read:

$$
\begin{aligned}
& Q_{n}\left[\Omega_{n+1}-\Omega_{n}\right]+\theta_{n+1}-\frac{R_{n}}{R_{n-1}} \theta_{n-1}=W Q_{n}^{\prime} \\
& \Omega_{n+1}+\Omega_{n}=-\frac{\theta_{n} Q_{n}}{R_{n}} \\
& \theta_{0}=U ; \quad \Omega_{0}=V ; \quad \theta_{-1}=0 .
\end{aligned}
$$

This system of equations reduces to:

$$
\begin{aligned}
& z^{2}\left(\gamma_{n+1}-\gamma_{n}\right)+\left(c_{n+1}-\frac{R_{n}}{R_{n-1}} c_{n-1}\right) z^{2}+\left(d_{n+1}-\frac{R_{n}}{R_{n-1}} d_{n-1}\right)=1 \\
& -4 \beta z^{3}+\left(\gamma_{n+1}+\gamma_{n}\right) z=-\frac{z}{R_{n}}\left(c_{n} z^{2}+d_{n}\right) \\
& \cdot c_{0} z^{2}+d_{0}=4 \beta z^{2}+1+4 \beta s_{2} \\
& -2 \beta z^{3}+\gamma_{0} z=-2 \beta z^{3}-\frac{z}{2} \\
& \cdot c_{-1} z^{2}+d_{-1}=0 .
\end{aligned}
$$

which by identification gives:

$$
\begin{array}{ll}
\gamma_{n+1}-\gamma_{n}+c_{n+1}-\frac{R_{n}}{R_{n-1}} c_{n-1}=0 & \text { when } n \geqq 1 \\
d_{n+1}=1+\frac{R_{n}}{R_{n-1}} d_{n-1} & n \geqq 0 \\
4 \beta=\frac{c_{n}}{R_{n}} & n \geqq 0 \\
\gamma_{n+1}+\gamma_{n}=-\frac{d_{n}}{R_{n}} & n \geqq 0 .
\end{array}
$$

The first relation in (IV.11) for $n=0$ reads differently:

$$
\gamma_{1}-\gamma_{0}+c_{1}=0
$$

To the set of Eqs. (IV.11) we have to add, the initial conditions:

$$
\begin{aligned}
& c_{0}=4 \beta \\
& d_{0}=1+4 \beta s_{2} \\
& \gamma_{0}=-1 / 2 \\
& R_{0}=1 \\
& c_{-1}=0 \\
& d_{-1}=0 .
\end{aligned}
$$


From (IV.13) we get

$$
\begin{aligned}
& c_{1}=4 \beta s_{2} \\
& d_{1}=1 \\
& \gamma_{1}=1 \\
& \gamma_{1}=-\left(\frac{1}{2}+4 \beta s_{2}\right) \\
& R_{1}=s_{2} .
\end{aligned}
$$

The value obtained for $R_{1}$ fits exactly the value in (II.18), which shows correctinitialization.

$$
\begin{aligned}
& \text { We shall rewrite (IV.11) as: } \\
& \gamma_{n+1}-\gamma_{n}=-4 \beta\left(R_{n+1}-R_{n}\right) \quad n \geqq 1 \\
& c_{n}=4 \beta R_{n} \\
& \gamma_{n+1}+\gamma_{n}=-\frac{d_{n}}{R_{n}} \\
& d_{n+1}=+\frac{R_{n}}{R_{n-1}} d_{n-1} .
\end{aligned}
$$

The first equation in (IV.15) is immediate to solve and gives:

$$
\gamma_{n}=\gamma_{1}+4 \beta R_{1}-4 \beta R_{n}=-\frac{1}{2}-4 \beta R_{n} .
$$

Finally we have:

$$
\begin{array}{ll}
\gamma_{n}=-\frac{1}{2}-4 \beta R_{n} & n \geqq 1 \\
c_{n}=4 \beta R_{n} & n \geqq 0 \\
d_{n}=R_{n}+4 \beta R_{n}\left(R_{n}+R_{n+1}\right) & n \geqq 1
\end{array}
$$

and

$$
\left[R_{n+1}-R_{n}\right]+4 \beta\left[R_{n+1}\left(R_{n+1}+R_{n+2}\right)-R_{n}\left(R_{n}+R_{n-1}\right)\right]=1 \quad n \geqq 2 .
$$

This last relation is the one we need to obtain the asymptotic expansion of $R_{k}(g / n)$, which will now be discussed.

Let us make a last remark, the relation (IV.8) holds only from $n=2$, because the last relation (IV.17) holds only from $n=1$. Therefore it is necessary to compute directly from (IV.15), $R_{2}$ and $R_{3}$ one gets:

$$
\begin{aligned}
& d_{2}=1+b_{2}+4 \beta s_{2}^{2} \\
& c_{2}=\frac{1-s_{2}-4 \beta s_{2}^{2}}{s_{2}} \\
& \gamma_{2}=\left(4 \beta s_{2}^{2}+\frac{1}{2} s_{2}-1\right) s_{2}^{-1} \\
& R_{2}=\frac{1-s_{2}-4 \beta s_{2}^{2}}{4 \beta s_{2}} \\
& c_{3}=\frac{12 \beta s_{2}^{2}+s_{2}-1}{s_{2}\left(1-s_{2}-4 \beta s_{2}^{2}\right)} \\
& d_{3}=\frac{1-s_{2}}{4 \beta s_{2}^{2}}
\end{aligned}
$$




$$
\begin{aligned}
\gamma_{3} & =\frac{1-\frac{3}{2} s_{2}+2\left(\frac{1}{4}-6 \beta\right) s_{2}^{2}+2 \beta s_{2}^{3}}{s_{2}\left(1-s_{2}-4 \beta s_{2}^{2}\right)} \\
R_{3} & =\frac{12 \beta s_{2}^{2}+s_{2}-1}{4 \beta s_{2}\left(1-s_{2}-4 \beta s_{2}^{2}\right)} .
\end{aligned}
$$

It is easy to check that the values of $R_{2}$ and $R_{3}$ obtained in this way are the same as the ones which one gets by making use of formulae (II.19), (II.21) and (III.4).

However if one sets $R_{0}=0$ in (IV.8) and $R_{1}=s_{2}$, the correct value of $R_{2}$ is then obtained, for $n=0$; then for $n=1$, also the correct value of $R_{3}$ is obtained. Therefore we can consider (IV.8) to be valid from $n=0$, provided we set $R_{0}=0$.

\section{Estimate of the Generating Functions $E_{0}(g)$ and $E_{1}(g)$}

To compute the generating functions $E_{0}(g)$ and $E_{1}(g)$ from formulae (III.14) and (III.15), it is necessary to have an asymptotic estimate of $\bar{R}_{p}(g / n)$.

When $g=0(\beta=0)$, Eq. (IV.18) reduces to:

$$
R_{n+1}(0)=1+R_{n}(0) \text {. }
$$

That is, using the previous remark, that in fact (V.1) is valid from $n=0$ with $R_{0}=0$ :

$$
R_{n}(0)=n \text {. }
$$

Therefore $\bar{R}_{k}=\frac{R_{k}(g / n)}{R_{k}(0)}$ satisfies the equation:

$$
\begin{gathered}
{\left[(k+1) \bar{R}_{k+1}-k \bar{R}_{k}\right]+\frac{4 g}{n}\left\{(k+1) \bar{R}_{k+1}\left[(k+1) \bar{R}_{k+1}+(k+2) \bar{R}_{k+2}\right]\right.} \\
\left.-k \bar{R}_{k}\left(k \bar{R}_{k}+(k-1) \bar{R}_{k-1}\right]\right\}=1 .
\end{gathered}
$$

Setting:

$$
\begin{aligned}
& x=\frac{p}{n} \\
& \varepsilon=\frac{1}{n} \\
& x \bar{R}_{n x}=y_{\varepsilon}(x)
\end{aligned}
$$

Eq. (V.3) becomes:

$$
\begin{aligned}
& {\left[y_{\varepsilon}[x+\varepsilon]-y_{\varepsilon}[x]\right]+4 g\left[y_{\varepsilon}(x+\varepsilon)\left[y_{\varepsilon}(x+\varepsilon)+y_{\varepsilon}(x+2 \varepsilon)\right]\right.} \\
& \left.-y_{\varepsilon}(x)\left[y_{\varepsilon}(x)+y_{\varepsilon}(x-\varepsilon)\right]\right]=\varepsilon .
\end{aligned}
$$

Expanding $y_{\varepsilon}(x)$ in powers of $\varepsilon$, we have:

$$
y_{\varepsilon}(x)=y(x)+\varepsilon y_{1}(x)+\varepsilon^{2} y_{2}(x)+\varepsilon^{3} y_{3}(x)+0\left(\varepsilon^{4}\right),
$$

and

$$
\begin{aligned}
y_{\varepsilon}(x+\varepsilon)= & y(x)+\varepsilon\left[y_{1}(x)+y^{\prime}(x)\right]+\varepsilon^{2}\left[y_{2}(x)+y_{1}^{\prime}(x)+\frac{1}{2} y^{\prime \prime}(x)\right] \\
& +\varepsilon^{3}\left[y_{3}(x)+y_{2}^{\prime}(x)+\frac{1}{2} y_{1}^{\prime \prime}(x)+\frac{1}{6} y^{\prime \prime \prime}(x)\right]+\ldots
\end{aligned}
$$




$$
\begin{aligned}
y_{\varepsilon}(x-\varepsilon)= & y(x)+\varepsilon\left[y_{1}(x)-y^{\prime}(x)\right]+\varepsilon^{2}\left[y_{2}(x)-y_{1}^{\prime}(x)+\frac{1}{2} y^{\prime \prime}(x)\right] \\
& +\varepsilon^{3}\left[y_{3}(x)-y_{2}^{\prime}(x)+\frac{1}{2} y_{1}^{\prime \prime}(x)-\frac{1}{6} y^{\prime \prime \prime}(x)\right]+\ldots \\
y_{\varepsilon}(x+2 \varepsilon)= & y(x)+\varepsilon\left[y_{1}(x)+2 y^{\prime}(x)\right]+\varepsilon^{2}\left[y_{2}(x)+2 y_{1}^{\prime}(x)+\frac{1}{2} y^{\prime \prime}(x)\right] \\
& +\varepsilon^{3}\left[y_{3}(x)+2 y_{2}^{\prime}(x)+2 y_{1}^{\prime \prime}(x)+\frac{4}{3} y^{\prime \prime \prime}(x)\right]+\ldots
\end{aligned}
$$

Identifying the powers in $\varepsilon$ in (V.5) we get, up to $\varepsilon^{2}$ terms:

$$
\begin{aligned}
& \varepsilon \text { term }: y^{\prime}(x)[1+24 g y(x)]=1 \\
& \varepsilon^{2} \text { term }:\left\{y^{\prime \prime}(x)[1+24 g y(x)]+24 g y^{\prime 2}(x)\right\}+2 y_{1}^{\prime}(x)+48 g\left[y(x) y_{1}(x)\right]^{\prime}=0 .(\text { V. } .9)
\end{aligned}
$$

Equation (V.9) simplifies, if one takes into account (V.8); it reduces to:

$$
\left[y_{1}(x)+24 g y(x) y_{1}(x)\right]^{\prime}=0,
$$

that is

$$
y_{1}(x)=K_{1} y^{\prime}(x) .
$$

However one has to take into account the boundary condition:

$$
y_{\varepsilon}(0)=0
$$

or

$$
y(0)=y_{1}(0)=y_{2}(0)=y_{3}(0)=\ldots=0 .
$$

Then integrating (V.8) we get:

$$
y(x)=\frac{-1+\sqrt{1+48 g x}}{24 g}
$$

and

$$
y_{1}(x)=0,
$$

taking into account (V.13).

The fact that $y_{1}(x)$ is identically zero simplifies the contribution of the $\varepsilon^{3}$ term, which reduces to:

$$
6 y_{2}^{\prime}+y^{\prime \prime \prime}+4 g\left\{y\left(36 y_{2}^{\prime}+12 y^{\prime \prime \prime}\right)+y^{\prime}\left(36 y_{2}+24 y^{\prime \prime}\right)\right\}=0 \text {. }
$$

However by differentiating twice equation (V.8), we can simplify (V.16), which becomes:

$$
\left[y_{2}+4 g\left[y y^{\prime \prime}+6 y y_{2}\right]\right]^{\prime}=0 ;
$$

or

$$
y_{2}=\left[K_{2}-4 g y y^{\prime \prime}\right] y^{\prime} .
$$

$K_{2}$ is fixed by the condition $y_{2}(0)=0$, which gives $K_{2}=0$

Finally

$$
y_{2}(x)=\frac{96 g^{2} y(x)}{[1+24 g y(x)]^{4}}=\frac{96 g^{2} y(x)}{(1+48 g x)^{2}} .
$$


And the corresponding functions $\rho(x)$ and $\rho_{2}(x)$ read:

$$
\begin{aligned}
& \rho(x)=\frac{y(x)}{x}=\frac{-1+\sqrt{1+48 g x}}{24 g x}, \\
& \rho_{2}(x)=\frac{y_{2}(x)}{x}=\frac{4 g}{x} \frac{(-1+\sqrt{1+48 g x)}}{(1+48 g x)^{2}} .
\end{aligned}
$$

As stated previously these functions are holomorphic in $x$ on the closed interval $[0,1]$ and strictly positive on this interval.

Formulae (V.20) and (V.21) give for $R_{p}(g / n)$ the expansion:

$$
\bar{R}_{p}(g / n)=\rho(p / n)+\frac{1}{n^{2}} \rho_{2}(p / n)+\ldots
$$

and

$$
\begin{aligned}
& \bar{R}_{0}(g / n)=1+\frac{96 g^{2}}{n^{2}}+\ldots \\
& \bar{R}_{1}(g / n)=1-\frac{12 g}{n}+384 \frac{g^{2}}{n^{2}}+\ldots \\
& \bar{R}_{2}(g / n)=1-\frac{24 g}{n}+1248 \frac{g^{2}}{n^{2}}+\ldots \\
& \bar{R}_{3}(g / n)=1-\frac{36 g}{n}+2688 \frac{g^{2}}{n^{2}}+\ldots
\end{aligned}
$$

$\bar{R}_{1}(g / n), \bar{R}_{2}(g / n)$ can be checked to agree up to the order $1 / n^{2}$, with the expansions one can get directly from formulae (IV.19), (IV.20) combined with (III.5).

Contrary to what we have stated in (III.10) we do not find $\bar{R}_{0}=1$, however the first formula of (V.23) shows clearly that the correction to the final formulae will be of order $1 / n^{3}$ which in the present situation is irrelevant.

Combining (V.20) and (III.14) we get, integrating by parts:

$$
\begin{aligned}
-E_{0}(g)= & \int_{0}^{1}(1-x) \log \rho(x) d x=\frac{1}{2} \log \frac{-1+\sqrt{1+48 g}}{24 g}-\int_{0}^{1}\left(x-\frac{x^{2}}{2}\right) \frac{\rho^{\prime}(x)}{\rho(x)} d x . \\
-E_{0}(g)= & \frac{1}{2} \log \frac{-1+\sqrt{1+48 g}}{24 g}+\frac{3}{8}-\frac{1}{2} \int_{0}^{1}(1+48 g x)^{-1 / 2} d x \\
& +\frac{1}{4} \int_{0}^{1} x(1+48 g x)^{-1 / 2} d x \\
= & \frac{1}{2} \log \frac{-1+\sqrt{1+48 g}}{24 g}+\frac{3}{8}+\frac{1}{48 g}+\frac{1}{3 \cdot(48 g)^{2}}-\frac{\sqrt{1+48 g}}{3 \cdot(48 g)^{2}}(1+120 g) .
\end{aligned}
$$

Setting

$$
a^{2}=\frac{-1+\sqrt{1+48 g}}{24 g}
$$


we recover formula of reference [1]:

$$
-E_{0}(g)=\frac{1}{2} \log a^{2}-\frac{1}{24}\left(a^{2}-1\right)\left(9-a^{2}\right)=\sum_{p=1}^{\infty}(-12 g)^{p} \frac{(2 p-1) !}{p !(p+2) !} .
$$

In the same way, combining (V.21) and (III.15) we get:

$$
-E_{1}(g)=-3 g+\frac{1}{12}\left[12 g-\log \frac{-1+\sqrt{1+48 g}}{24 g}\right]+96 g^{2} \int_{0}^{1} \frac{(1-x) d x}{(1+48 g x)^{2}}
$$

or

$$
-E_{1}(g)=-\frac{1}{12} \log \frac{1+48 g-\sqrt{1+48 g}}{24 g}=\frac{1}{24} \sum_{p=1}^{\infty} \frac{(-12 g)^{p}}{p}\left[4^{p}-\frac{(2 p) !}{(p !)^{2}}\right]
$$

The first coefficients of the expansion of $E_{1}(g)$ are:

$$
E_{1}(g)=g-30 g^{2}+1056 g^{3}-\ldots
$$

Using the variable $a^{2}$ defined in (V.27) we get:

$$
E_{1}\left(a^{2}\right)=\frac{1}{12} \log \left(2-a^{2}\right) \text {. }
$$

Acknowledgements. The author thanks Dr. C. Itzykson for having brought to his attention this problem, as well as Dr. J. B. Zuber for comments, and Dr. J. Zınn Justin for a careful readıng of the manuscript. He also acknowledges Dr. B. Derrida for a fruitful discussion.

Note Added in Proof. Dr. J. B. Zuber has worked out the $E_{2}$ function:

$$
E_{2}(g)=\frac{1}{6 !} \frac{\left(1-a^{2}\right)^{3}}{\left(2-a^{2}\right)^{5}}\left(82+21 a^{2}-3 a^{4}\right) \text {. }
$$

\section{Appendix I}

We want to prove formula (II.3). From the definition of a determinant we have:

$$
\begin{aligned}
& D_{n-1}=\sum(-)^{P\left(i_{1}, i_{2}, \ldots i_{n}\right)} \mu_{-1+i_{1}} \mu_{i_{2}} \mu_{1+i_{3}} \ldots \mu_{(n-2)+i_{n}} \\
& =\int_{-\infty}^{+\infty} \sum_{j=1}^{j=n} d \mu\left(x_{j}\right) \sum(-)^{P\left(i_{1}, i_{2}, \ldots i_{n}\right)} x_{1}^{-1+i_{1}} x_{2}^{i_{2}} x_{3}^{1+i_{3}} \ldots x_{n}^{(n-2)+i_{n}} \\
& =\int_{-\infty}^{+\infty} \sum_{j=1}^{j=n} d \mu\left(x_{j}\right) x_{1}^{0} x_{2}^{1} x_{3}^{2} \ldots x_{n}^{n-1}\left|\begin{array}{cccc}
1 & 1 & \ldots & 1 \\
x_{1} & x_{2} & x_{n} \\
\ldots \ldots \ldots & \ldots \ldots \ldots \ldots . \\
x_{1}^{n-1} & x_{2}^{n-1} & x_{n}^{n-1}
\end{array}\right| .
\end{aligned}
$$

If one permutes in (AI.3) the $x_{j}$ in all possible manners and adds up the results, one gets:

which is just (AI.3).

$$
n ! D_{n-1}=\int_{-\infty}^{+\infty} \sum_{j=1}^{j=n} d \mu\left(x_{j}\right)\left|\begin{array}{cccc}
1 & 1 & \ldots & 1 \\
x_{1} & x_{2} & \ldots & x_{n} \\
x_{1}^{n-1} & & & x_{n}^{n-1}
\end{array}\right|^{2}
$$




\section{Appendix II}

The Laguerre method.

We shall, for completeness explain in detail the Laguerre method for expanding in continued fraction, the solution of a linear differential equation of the first order with polynomial coefficients.

$\bar{G}(z)$, the generating function of the moments is the solution of:

$$
W(z) \bar{G}^{\prime}(z)=2 V(z) \bar{G}(z)+U(z),
$$

where

$$
\begin{aligned}
& W(z)=1 \\
& V(z)=-\frac{z}{2}-2 \beta z^{3} \\
& U(z)=\left(1+4 \beta s_{2}\right)+4 \beta z^{2}
\end{aligned}
$$

and

$$
\bar{G}(z)=\frac{s_{0}}{z}+\frac{s_{1}}{z^{2}}+\ldots \frac{s_{k}}{z^{k+1}}+\ldots
$$

with

$$
\begin{aligned}
& s_{0}=1 \\
& s_{2 k+1}=0 .
\end{aligned}
$$

We see that $\bar{G}(z)$ is formally an odd function of $z$. Therefore the approximation

$$
[n-1 / n](z)=\frac{N_{n}(z)}{M_{n}(z)},
$$

has its polynomials $M_{n}(z)$ which are of parity $(-)^{n}$ and degree $n$ while $N_{n}(z)$ are of parity $(-)^{n-1}$ and degree $n-1$.

We have by definition of the $[n-1 / n]$ Pade Approximations:

$$
\bar{G}(z)=\frac{N_{n}(z)}{M_{n}(z)}+0\left(z^{-(2 n+1)}\right) .
$$

If we derive with respect to $z$, we get:

$$
\bar{G}^{\prime}(z)=\frac{N_{z}^{\prime}(z) M_{n}(z)-M_{n}^{\prime}(z) N_{n}(z)}{M_{n}^{2}(z)}+0\left(z^{-(2 n+2)}\right)
$$

and introducing (AII.7) into (AII.1):

$$
U(z) M_{n}^{2}(z)+2 V(z) N_{n}(z) M_{n}(z)-W(z)\left[N_{n}^{\prime}(z) M_{n}(z)-M_{n}^{\prime}(z) N_{n}(z)\right]=A_{n} \theta_{n}(z),
$$

where $A_{n}$ is a constant suitably chosen and $\theta_{n}(z)$ a polynomial in $z$ such that:

$$
A_{n} \theta_{n}(z)=M_{n}^{2}(z)\left\{W(z) \cdot 0\left(z^{-(2 n+2)}\right)+V(z) \cdot 0\left(z^{-/ 2 n+1)}\right)\right.
$$


(AII.9) shows clearly that $\theta_{n}(z)$ is at most of degree 2 in $z$, taking into account that $W(z)$ is of degree zero and $V(z)$ of degree 3.

Therefore we can set:

$$
\theta_{n}(z)=c_{n} z^{2}+d_{n} \text {. }
$$

There is no odd term in $\theta_{n}(z)$ by the parity argument.

We recall that the polynomials $M_{n}(z)$ and $N_{n}(z)$ fulfil: [5]

$$
N_{n+1}(z) M_{n}(z)-M_{n+1}(z) N_{n}(z)=A_{n+1} .
$$

where we fix the previous constant which was arbitrary to be equal to the constant in (AII.11).

Pluging the value of $A_{n}$ from (AII.11) into (AII.8), we get:

$$
\begin{aligned}
& {\left[U(z) M_{n}(z)+V(z) N_{n}(z)-W(z) N_{n}^{\prime}(z)+\theta_{n}(z) N_{n-1}(z)\right] M_{n}(z)} \\
& \quad=\left[\theta_{n}(z) M_{n-1}(z)-W(z) M_{n}^{\prime}(z)-V(z) M_{n}(z)\right] N_{n}(z),
\end{aligned}
$$

which implies due to the fact that $\frac{N_{n}(z)}{M_{n}(z)}$ is an irreducible fraction ${ }^{2}$ :

$$
\begin{aligned}
& W(z) M_{n}(z)=\left[\Omega_{n}(z)-V(z)\right] M_{n}(z)+\theta_{n}(z) M_{n-1}(z) \\
& W(z) N_{n}^{\prime}(z)=\left[\Omega_{n}(z)+V(z)\right] N_{n}(z)+\theta_{n}(z) N_{n-1}(z)+U(z) M_{n}(z)
\end{aligned}
$$

where $\Omega_{n}(z)$ is a polynomial.

From (AII.11) written for $A_{n}$ and $A_{n+1}$, one gets:

$$
\left[A_{n} N_{n+1}(z)+A_{n+1} N_{n-1}(z)\right] M_{n}(z)=\left[A_{n} M_{n+1}(z)+A_{n+1} M_{n-1}(z)\right] N_{n}(z) .
$$

Setting

$$
R_{n}=\frac{A_{n+1}}{A_{n}}
$$

we see, using again the irreducibility of $\frac{N_{n}(z)}{M_{n}(z)}$ that (AII.15) implies:

$$
\begin{aligned}
& N_{n+1}(z)-Q_{n}(z) N_{n}(z)+R_{n} N_{n-1}(z)=0, \\
& M_{n+1}(z)-Q_{n}(z) M_{n}(z)+R_{n} M_{n-1}(z)=0,
\end{aligned}
$$

where $Q_{n}$ is a polynomial of degree 1 , which by parity has no constant term, furthermore here:

$$
Q_{n}(z)=z,
$$

because our polynomials are normalized following (II.12) and (II.13).

We have now to fix the degree of $\Omega_{n}(z)$. From (AII.13), one gets:

$$
\Omega_{n}(z)=V(z)+W(z) \frac{M_{n}^{\prime}(z)}{M_{n}(z)}-\theta_{n}(z) \frac{M_{n-1}(z)}{M_{n}(z)} .
$$

Sending $z \rightarrow+\infty$, we see that $\Omega_{n}(z)$ increases like the term of highest degree of the

2 This is so, because the zeros of $N_{n}(z)$ are separated by those of $M_{n}(z)[5]$ 
polynomial part of:

$$
V(z)+n \frac{W(z)}{z},
$$

that is, here:

$$
\Omega_{n}=-2 \beta z^{3}+\gamma_{n} z .
$$

There is no even part in $\Omega_{n}$ by parity argument.

If we derive (AII.18) and multiply by $W(z)$, we get:

$$
W(z) M_{n+1}^{\prime}(z)-W(z) Q_{n}(z) M_{n}^{\prime}(z)-W(z) Q_{n}^{\prime}(z) M_{n}(z)+W(z) R_{n} M_{n-1}^{\prime}(z)=0 .
$$

But using (AII.13) written for $n-1, n$ and $n+1$, in (AII.23) we obtain:

$$
\begin{aligned}
& \left(\Omega_{n+1}-V\right) M_{n+1}+\left[\theta_{n+1}-W Q_{n}^{\prime}-Q_{n}\left(\Omega_{n}-V\right)\right] M_{n} \\
& \quad+\left[R_{n}\left(\Omega_{n-1}-V\right)-Q_{n} \theta_{n}\right] M_{n-1}+R_{n} \theta_{n-1} M_{n-2}=0 .
\end{aligned}
$$

Substituting

$$
M_{n+1}=Q_{n} M_{n}-R_{n} M_{n-1},
$$

we get:

$$
\begin{aligned}
& {\left[Q_{n}\left(\Omega_{n+1}-\Omega_{n}\right)-Q Q_{n}^{\prime}+\theta_{n+1}\right] M_{n}-\left[R_{n}\left(\Omega_{n+1}-\Omega_{n-1}\right)+Q_{n} \theta_{n}\right] M_{n-1}} \\
& \quad+R_{n} \theta_{n-1} M_{n-2}=0 .
\end{aligned}
$$

This Eq. (AII.26) must be compared with (AII.18) written for $(n-1)$. And therefore:

$$
\begin{aligned}
& Q_{n}(z)\left(\Omega_{n+1}(z)-\Omega_{n}(z)\right)+\theta_{n+1}(z)-\frac{R_{n}}{R_{n-1}} \theta_{n-1}(z)=W(z) Q_{n}^{\prime}(z) . \\
& R_{n}\left(\Omega_{n+1}(z)-\Omega_{n-1}(z)\right)=\frac{R_{n}}{R_{n-1}} Q_{n-1}(z) \theta_{n-1}(z)-Q_{n}(z) \theta_{n}(z)
\end{aligned}
$$

By adding up all Eqs. (AII.28) we get:

$$
\left(\Omega_{n+1}+\Omega_{n}\right)=-\frac{\theta_{n} Q_{n}}{R_{n}}+\left[\left(\Omega_{-1}+\Omega_{0}\right)+\frac{Q_{-1} \theta_{-1}}{R_{-1}}\right] .
$$

But initialization implies:

$$
\begin{aligned}
& \theta_{0}(z) \equiv U \\
& \theta_{-1}(z) \equiv 0 \\
& \Omega_{0}(z) \equiv V(z) \\
& \Omega_{-1}(z) \equiv-V(z),
\end{aligned}
$$

and therefore

$$
\left(\Omega_{n+1}+\Omega_{n}\right)=-\frac{\theta_{n} Q_{n}}{R_{n}} .
$$

All the necessary machinery for section IV has been set up. 


\section{Refrences}

1. Brezin, E., Itzykson, C., Parisi, G., Zuber, J. B. : Planar diagrams. Commun. Math. Phys. 59, 3551 (1978)

2. Akhiezer, N. I. : The classical moment problem, p. 23. Oliver and Boyd 1965

3. Same as Ref. $[2$, p. 5]

4. Laguerre, E. : Sur la réduction en fractions continues d'une fonction qui satisfait à une équation différentielle linéaire du premier ordre dont les coefficients sont rationnels. J. Math. 1, 135-165 (1885)

5. Same as Ref. $[2$, pp. 9, 10]

Communicated by R. Stora

Received February 28, 1979 
